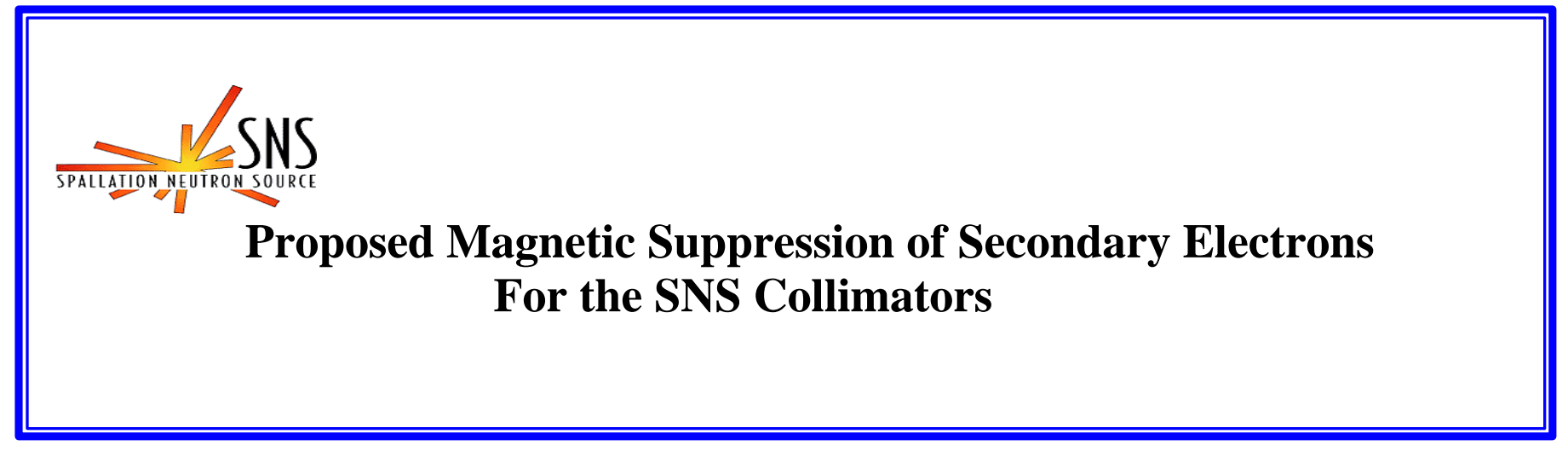

BNL/SNS TECHNICAL NOTE

NO. 057

P. Thieberger

February 8, 1999

ALTERNATING GRADIENT SYNCHROTRON DEPARTMENT BROOKHAVEN NATIONAL LABORATORY UPTON, NEW YORK 11973 


\title{
Proposed Magnetic Suppression of Secondary Electrons for the SNS Collimators
}

\author{
P. Thieberger
}

\section{Introduction}

Possible deleterious effects due to electron-proton (e-p) instabilities in synchrotron and storage rings have been considered for the Bevatron [1], the ISR [2], the PSR [3], and the proposed Spallation Neutron Source (SNS) [4]. In view of the excellent vacuum and large vacuum chambers planned for the SNS, the most likely and abundant source of electrons in this machine will be the grazing incidence of protons on the interior surfaces of the collimators. Here we suggest the introduction of a weak axial magnetic field at the

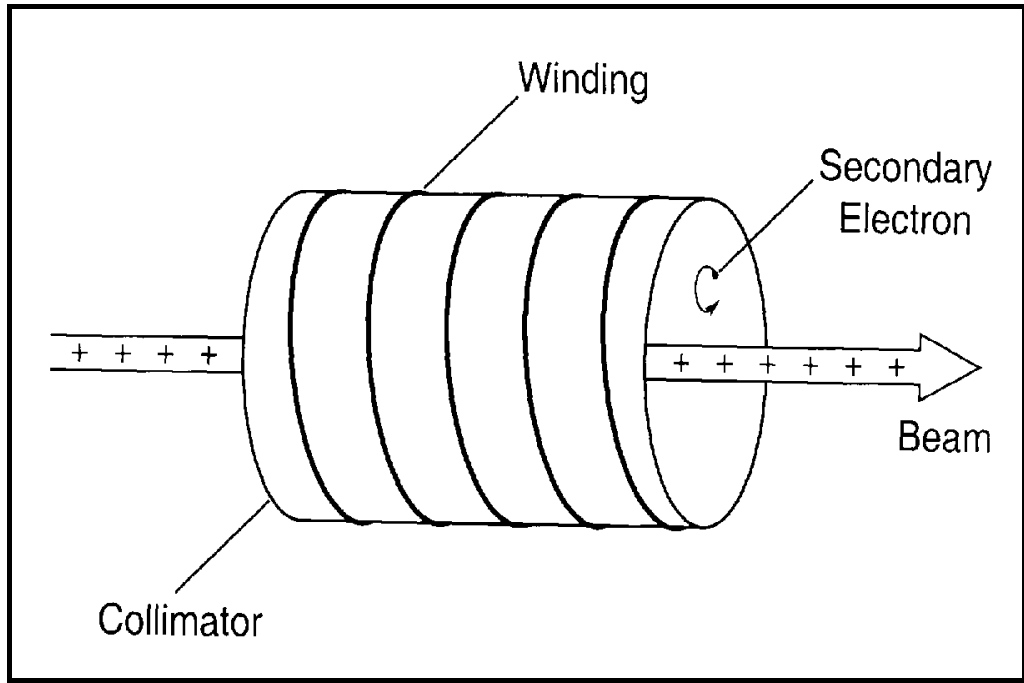

Fig. 1 Secondary electrons originating at the interior wall of the collimator rapidly return to the wall due to the presence of a longitudinal magnetic field produced by current in a solenoidal winding. location of the SNS collimators to effectively suppress these secondary electrons before they can reach the proton beam. Such a field could be generated by solenoidal windings built into the collimators. The relatively slow secondary electrons (energy typically $<100 \mathrm{eV}$ ) would then follow trajectories that would rapidly return the vast majority of them to the surface of the collimator, even in the presence of rather intense radial accelerating fields due to the intense positive proton beam (fig.1).

The suppression of unwanted electrons with relatively weak magnetic fields is widely used, for example, to avoid false readings in Faraday cups used to measure ion beam intensities, to suppress intense electron beams emanating from negative ion sources and in electrostatic accelerators, either alone or in conjunction with inclined fields, to reduce acceleration tube vacuum breakdowns caused by cascading electrons [5].

Here we review some of the relevant equations and show some quantitative examples which should enable us to estimate the magnitude of the required magnetic field. At the end we suggest some simple demonstration experiments. 


\section{Electron Trajectories}

Let's start by writing down the cyclotron frequency $\omega$ and the orbit radius $r$ for an electron in a uniform magnetic field B. In Gaussian cgs units:

$\omega=(\mathrm{e} B) /(\mathrm{m} \mathrm{c})$

and

$\mathrm{r}=\mathrm{v \perp} / \omega$

where $\mathrm{v} \perp$ is the component of the velocity perpendicular to the field.

A secondary electron ejected tangentially to a surface will thus reach a maximum distance D from the surface given by:

$\mathrm{D}=2 \mathrm{v} \perp / \omega$

As an example we shall consider a $100 \mathrm{eV}$ electron moving across a 100 Gauss field:

$\mathrm{e}=4.8 \times 10^{-10} \mathrm{esu}$

$\mathrm{m}=9.1 \times 10^{-28} \mathrm{~g}$

$\mathrm{c}=3 \times 10^{10} \mathrm{~cm} / \mathrm{s}$

$\mathrm{B}=100$ Gauss

We get from 1) above:

$\omega=1.76 \times 10^{9} 1 / \mathrm{s}$ for the angular velocity

and

$v=\omega / 2 \pi=2.8 \times 10^{8} 1 / \mathrm{s}=280 \mathrm{MHZ}$ for the rotational frequency

The velocity v of a $100 \mathrm{eV}$ electron can be obtained from:

$511 \mathrm{KeV}+100 \mathrm{eV}=511 \mathrm{KeV} /\left(1-\mathrm{v}^{2} / \mathrm{c}^{2}\right) \quad$ where $511 \mathrm{KeV}$ is the energy equivalent of the electron rest mass. We obtain:

$\mathrm{v}=5.9 \times 10^{8} \mathrm{~cm} / \mathrm{s} \quad(\beta \approx 0.02)$

If $\mathrm{v}$ is perpendicular to the field, then we get from (3) above 
$\mathrm{D}=2 \times\left(5.9 \times 10^{8} \mathrm{~cm} / \mathrm{s}\right) /\left(1.76 \times 10^{9} 1 / \mathrm{s}\right)=0.67 \mathrm{~cm}$

for the maximum distance reached from the surface by a $100 \mathrm{eV}$ electron ejected tangentially to that surface and at 90 degrees to a 100 Gauss magnetic field.

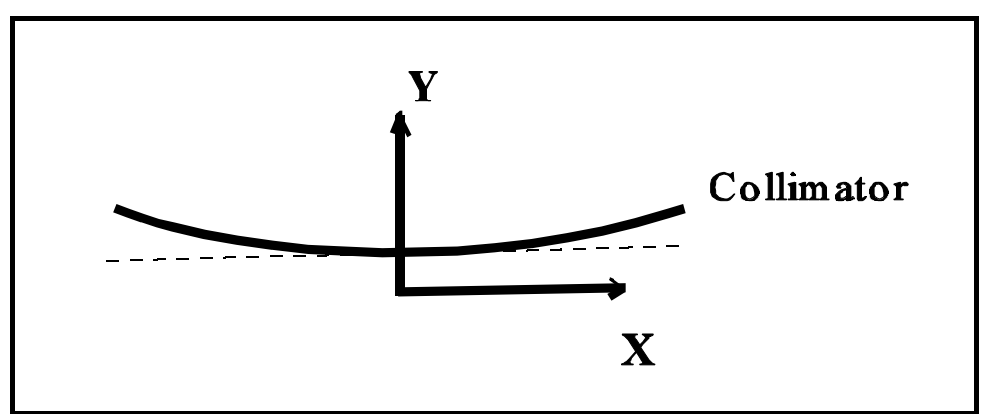

Fig.2 Schematic representation of part of collimator wall section, showing the relative position of the coordinate axes used below.
Now we will consider what happens if we add a uniform electric field $\mathrm{E}_{\mathrm{y}}$ perpendicular to $\mathrm{B}_{\mathrm{z}}$. This will be a reasonable approximation to the cylindrical geometry with the electric field created by the presence of the positive beam, as long as we consider only electron trajectories confined to the close vicinity of the collimator surface. This condition is required for good electron suppression and shouldn't be too difficult to achieve in view of the above example which shows a radius of curvature of the trajectory that is much smaller than the radius of the collimator for a rather modest magnetic field. Exact electron trajectories in this cylindrical "inverted magnetron" geometry of the collimator could of course also be calculated, but this is complicated and not necessary for the time being.

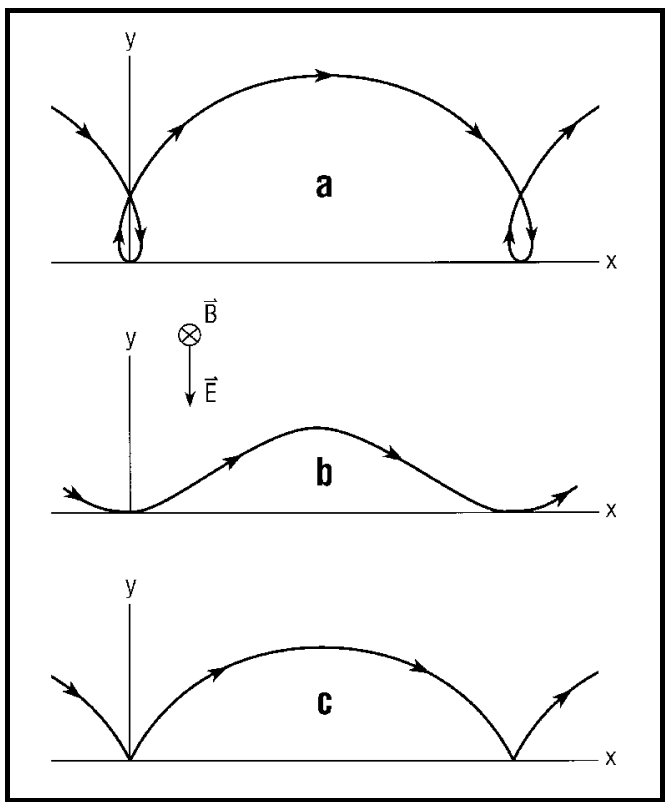

Fig. 3 Trochoid electron trajectories from ref. 6 for different values of the parameter a (see text).
Following Landau and Lifshitz [6], we write the parametric equations of the electron trajectory projection on the xy plane perpendicular to the beam and to the magnetic field:

$x=(a / \omega) \sin \omega t+\left(c E_{y} / B_{z}\right) t$

$y=(\mathbf{a} / \omega)(\cos \omega t-1)$

where $\omega$ is given by (1), the $\mathrm{z}$ axis is parallel to the beam, the $y$ axis is perpendicular to the collimator surface and $x$ is parallel to the tangent to that surface (fig.2). The parameter $\mathbf{a}$ as well as the offset of the origin with respect to the collimator surface depend on the initial electron velocity and angle of emission.

Equations (4) and (5) define trochoids such as the ones shown in Figs. 3a, 3b, and 3c for values of the parameter $\mathbf{a}>,<$, or $=\mathrm{c} \mathrm{E}_{\mathrm{y}} / \mathrm{B}_{\mathrm{z}}$, respectively. 
Secondary electrons will have continuous energy and angular distributions. For a given energy it is easy to see that the worst case in terms of the trajectory extending furthest along the y-axis is an electron leaving tangentially to the surface in a direction such as indicated at the xy origin in fig 3a. In this special case the y-offset in Fig. 2 disappears.

At $\mathrm{t}=0$ we get from (4) and (5):

$\mathrm{dy} / \mathrm{dt}=0$

$\mathrm{dx} / \mathrm{dt}=\mathbf{a}+{ }_{\mathrm{y}} / \mathrm{B}_{\mathrm{z}}$

For $\mathrm{E}_{\mathrm{y}}=0$, the trajectory described by (4) and (5) is, of course, a circle. From (7) we get: $\mathbf{a}=\mathrm{dx} / \mathrm{dt}=\mathrm{v} \perp, \quad$ and $\mathbf{a} / \omega=\mathrm{v} \perp / \omega$ is the radius as before (2), and $\mathrm{D}=2 \mathrm{v} \perp / \omega \quad$ (3) is the maximum excursion.

For $\mathrm{E}_{\mathrm{y}} \neq 0$, the absolute value of a obtained from (7) (depending on the sign of the different quantities) can be as large as:

$\mathbf{a}=\mathrm{v} \perp+\quad{ }_{\mathrm{y}} / \mathrm{B}_{\mathrm{z}}$

and from (6) we get:

$\mathrm{D}=2 \times\left(\mathrm{v} \perp+\quad{ }_{\mathrm{y}} / \mathrm{B}_{\mathrm{z}}\right) / \omega$

If we now add an electric field of 1 esu (300 Volts/cm) to our previous example we have:

$\mathrm{V} \perp=5.9 \times 10^{8} \mathrm{~cm} / \mathrm{s} \quad$ (as before)

$$
{ }_{\mathrm{y}} / \mathrm{B}_{\mathrm{z}}=3 \times 10^{10} \times 1 \text { esu } / 100 \text { Gauss }=3 \times 10^{8} \mathrm{~cm} / \mathrm{s}
$$

from (9) we get $\mathrm{D}=1.01 \mathrm{~cm}$ for the maximum y-excursion for a $100 \mathrm{eV}$ electron ejected tangentially to a surface, at 90 degrees to a 100 Gauss magnetic field which is parallel to the surface in the presence of a $300 \mathrm{Volt} / \mathrm{cm}$ accelerating field perpendicular to the surface. 


\section{Demonstration Experiments}

Simple experimental setups to test these ideas are schematically shown in figs. 4 and 5. Beams from the BNL Tandem would be used to generate the secondary electrons at the internal surface of a negatively biased cone or cylinder,

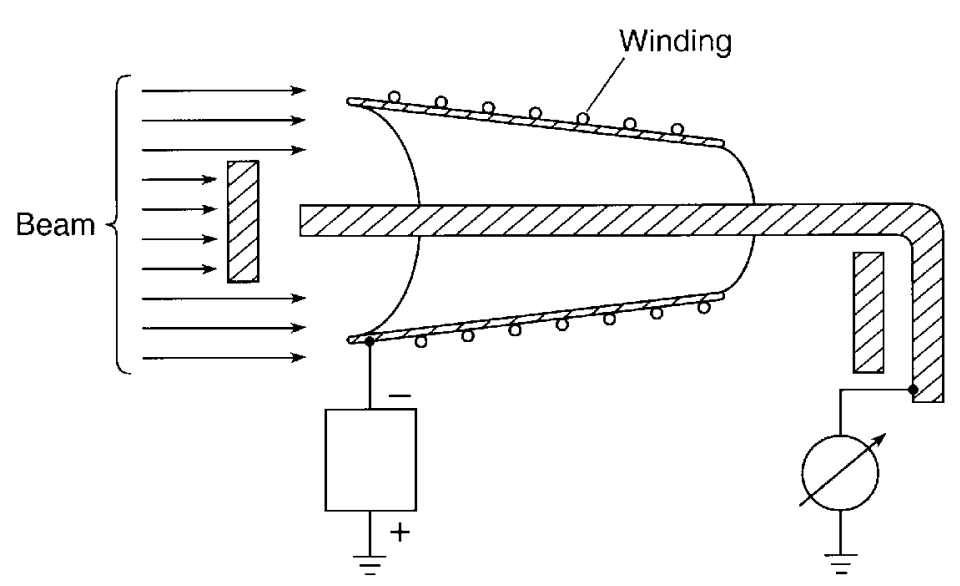

Fig. 4 A wide uniform beam impinges upon the interior of a conical electrode with appropriately placed beam stops to prevent any beam from hitting the central electrode.

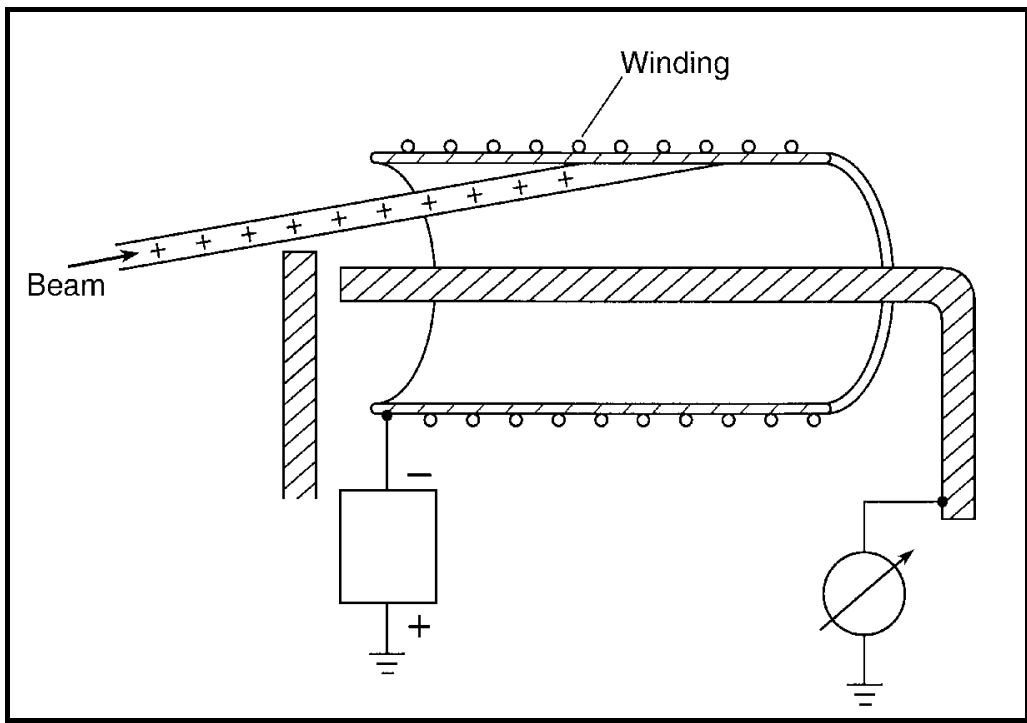

Fig. 5 A pencil-like beam hits a spot on the interior surface of a cylindrical electrode. The central electrode is used to measure the flow of secondary electrons as a function of the electron-suppressing magnetic field generated by a current in the winding and the potentials that will be generated by the intense SNS proton beam would be simulated by a current sensing electrode close to ground potential. The secondary electron current intensity would then be measured as a function of the magnetic field created by a variable current in the winding.

The beam current and therefore the secondary electron signal will be much smaller for the second setup. However, if the signal is sufficiently large for an accurate measurement, this would be the preferred configuration because the geometry is more realistic and because hardware limitations restrict beam widths to a maximum of about 2" for existing beam lines at the BNL Tandem. 


\section{Conclusions}

It was shown that relatively weak axial magnetic fields should be effective in causing most secondary electrons generated at the SNS collimators to rapidly return to the collimator surface. The suggested demonstration experiments would help to validate this approach.

Questions that remain to be answered include the degree to which these weak magnetic fields will affect the SNS proton beam, especially in view of the fact that they are of course not strictly axial except at the center of the collimators. Also the importance of suppressing the secondary electrons remains controversial.

\section{Acknowledgement}

I would like to gratefully acknowledge advice, encouragement and useful information received from Hans Ludewig.

\section{References}

1) H.A. Grunder and G.R. Lambertson, "Traverse Beam Instabilities at the Bevatron" , Proc. $8^{\text {th }}$ Int. Conf. on High Energy Accelerators. CERN Switzerland (1971)

2) E. Keil and B. Zotter, "Landau-damping of coupled E-P oscillations", CERN Report CERNISR-TH/71-58 (1971)

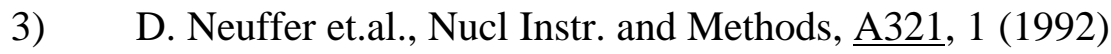

4) A.G. Ruggiero and M. Blaskiewicz, "E-P Instability in the NSNS Accumulator Ring", Particle Accelerator Conf., Vancouver, B.C., Canada. (1997)

5) P. Thieberger, Nuclear Instrument \& Methods 150 (1978) 327.

6) Landau and Lifshitz, The Classical Theory of Fields, Addison-Wesley (1951) 